\title{
Liquid-phase adsorption: common problems and how we could do better
}

\author{
Nikolay Cherkasov a,b \\ a School of Engineering, University of Warwick, Coventry CV4 7AL, United Kingdom \\ b Stoli Catalysts Ltd, Coventry, CV3 4DS, UK \\ corresponding author: n.cherkasov@warwick.ac.uk
}

\section{Abstract}

Adsorption plays a vital role in many applications from adsorbents for concentrating valuable compounds or removing pollutants to catalysts. In gas and liquid phases, the adsorption phenomena may look similar and the results are often transferred. But solvents play a role and may change the adsorption behaviour even for strong adsorbates - liquid phase adsorption is different!

The review covers kinetics and thermodynamics of adsorption processes and focuses on several areas that receive only minor attention despite being crucial for obtaining reliable results. Such underappreciated areas include the analysis of how to maximise experimental accuracy of adsorption studies and analyse the model parameters and their confidence intervals; the effect of the mathematical representation and model linearization on the results; the possibility of processes other than adsorption during the experiments.

The experiments based on disappearance of the adsorbate from the equilibrium solution shall be performed to ensure at least $10 \%$ decrease in concentration for reasonable accuracy. Regression analysis and analysis of the confidence intervals of the parameters merit particular attention as well as an independent validation of the model assumptions. Even an excellent data fit may provide results differing by several times from the correct values.

Adding to the dispute on dimensions in the adsorption constants in van't Hoof equation, the review adds arguments in favour of using constants in L/mol units. The review concludes with the proposed workflow in the analysis of liquid-phase adsorption data from the data acquisition to data analysis and modelling and offers a Matlab app for Langmuir adsorption data analysis.

Keywords: adsorption; error; Langmuir; catalysis; Equilibrium constant; adsorption kinetics 


\section{Introduction}

In the United Kingdom as an example, chemicals constitute the $3^{\text {rd }}$ largest manufacturing sector that employs 400,000 people and generates $£ 48$.7bn turnover [1]. The chemicals manufacturing relies on catalytic processes, mainly heterogeneous catalysis, judging by the value and scale of the production [2,3]. There is barely a synthesis that does not include a catalytic step - from petroleum cracking into fuels to the synthesis of cancer drug candidates [4-6]. Adsorption extends far beyond catalysis being vital in pollution removal, in concentrating of rare compounds. Aqueous effluents from most industries such as paint, pesticide, petroleum, printing, pharmaceutics, wood, paper, steel, textile contain chemicals are treated by adsorption processes [7].

Adsorption of substrates on the catalyst surface is one of the key reaction steps - the steps critical for the catalyst performance but all too often overlooked. Adsorption energy must fall within a narrow range to provide the highest reaction rate - too strong adsorption hinders reaction creating catalyst surface overly occupied with the substrate, too weak interaction provides low catalyst coverage and low probability of the reaction. This Sabatier principle is one of the key findings in catalysis - the corresponding volcano plots (reaction rate versus adsorption energy) are observed in all the areas of catalysis [8-10]. Complex reaction networks with multiple intermediates and bond formations could be accurately described only in terms of a single desorption process [11]. On the other hand, the adsorption phenomena are sometimes detrimental for catalysis - strong adsorption of by-products is often the cause for catalyst deactivation [12-15]. Hence, understanding of the adsorption processes is vital for the catalyst design.

The adsorption process itself, however, is scarcely studied directly in liquid-phase reactions. The insights are often obtained only indirectly using kinetic modelling or DFT computation. Data generated in gas-phase is often transferred into the liquid phase. This approach seems reasonable especially for non-reactive solvents and strong adsorbates. However, such a common sense does not always hold. For example, Pt nanoparticles encapsulated with polymer dendrimers show drastically different behaviour in solvents compared to gas-phase even for carbon monoxide [16]. In gas phase, the particles adsorb carbon monoxide weakly with low capacity; in liquid-phase, both the capacity and adsorption energy increase because the dendrimer polymer swells and opens the catalyst surface [16]. Considering that such a dramatic effect was observed for one of the strongest adsorbates, the validity of a casual transfer of results from gas phase into the liquid phase becomes questionable.

The study of liquid-phase adsorption in catalysts differs from conventional adsorbates, and this difference may bring insights into the conventional adsorption. Conventionally, the dyes, 
heavy metals or environmental pollutants in low concentration are adsorbed over active carbons or natural materials [7,17-20]. By their design and intended application, these adsorbents must have a high adsorption capacity with high adsorption constant. For catalysts, in contrast, the Sabatier principle limits the adsorption constant. Moreover, the supported catalysts with a few percent of the active component have a much lower adsorption capacity. Both the lower constant and capacity make the adsorption studies much more challenging and demanding. Such demands, however, surface many critical aspects of the adsorption studies; therefore, the insights and conclusions derived will be of value for all aspects of the liquid phase adsorption.

Understanding the adsorption processes with direct studies in liquid phase could open ways for a deeper understanding of the reaction mechanisms and development of more selective and efficient catalysts. This review discusses the main adsorption models and potential problems which attract little attention but can have drastic implications on the results obtained.

\section{Experiments on liquid-phase adsorption}

The adsorption experiments are generally based on the material balance. An adsorbent material is exposed to the adsorbate solution with a known concentration. The adsorbate concentration decreases in the solution $\left(V_{\text {solution }}\right)$ and adsorption $\left(q_{e}\right)$ is calculated as the difference between its introduced $\left(C_{\text {initial }}\right)$ and the remaining concentrations $\left(C_{\text {equilibrium }}\right)$ based on the equation 1. Adsorption is often normalised by the adsorbent mass.

$q_{e}=V_{\text {solution }}\left(C_{\text {initial }}-C_{\text {equilibrium }}\right)$.

This approach is widely used in the analysis of dyes or metal ions [17,21-24] as well as for the catalytic applications [25]. Obviously, this material balance approach is simple, however, there are several problems that the reader must be aware of.

The first problem with the approach is the possibility of unforeseen interactions. These may include chemical reactions, reactions with the impurities, and the effect of the adsorbent on the medium. For example, the adsorption of $\mathrm{Cr}(\mathrm{VI})$ brought in contact with an adsorbent may lead to reduction in addition to adsorption. Species of $\mathrm{Cr}(\mathrm{VI})$ may react with the surface to form $\mathrm{Cr}$ (III) and be desorbed [26]. As a result of $\mathrm{Cr}$ (III) desorption, the $\mathrm{Cr}(\mathrm{VI})$ content in the solution decreases. In the case of photometric analysis of $\mathrm{Cr}(\mathrm{VI})$ concentration, a strong decrease will be observed that may be wrongly attributed to high adsorption. Hence to avoid such problems, a possibility of such reactions as well as alternative analysis methods shall be considered. 
92 The second problem comes from using the concentration difference in equation 1 which may

93 dramatically increase measurement errors. Equation 2 shows the error in the adsorption

94 determined with the experimental errors denoted with symbol $\delta$ :

$95 \delta q_{e}=\left|q_{e}\right| \sqrt{\frac{\delta V^{2}}{V^{2}}+\frac{\delta C_{\text {initial }}^{2}+\delta C_{\text {equilibrium }}^{2}}{\left(C_{\text {initial }}-C_{\text {equilibrium }}\right)^{2}}}$.

96 The second term under the square root shows that the error in the adsorption increases

97 when the relative change in concentration is small. This conclusion is intuitive - analysis of

98 small changes against a large background is difficult.

99 The analytical error (in concentration) is often proportional to the determined value (

$100 \delta C=\xi \cdot C)$ when concentration is far from their detection limits [27,28]. Neglecting errors in

101 volume measurement, the relative error in adsorption may be simplified as shown in

102 equation 3 :

103

$\frac{\delta q_{e}}{\left|q_{e}\right|}=\xi \frac{\sqrt{C_{\text {initial }}^{2}+C_{\text {equilibrium }}^{2}}}{\left|C_{\text {initial }}-C_{\text {equilibrium }}\right|}=\xi \sqrt{1-\frac{2}{\Delta C_{\text {relative }}}+\frac{2}{\Delta C_{\text {relative }}^{2}}}$,

104 where $\Delta C_{\text {relative }}=\frac{C_{\text {initial }}-C_{\text {equilibrium }}}{C_{\text {initial }}}$.

105 Fig. 1 shows that the error in the adsorption analysis is affected by the relative decrease in 106 concentration. If the relative change in concentration is small $(<0.05)$, the error in adsorption 107 dramatically increases rendering results unreliable. A relative change in concentration, 108 however, depends on both the intrinsic adsorption properties and on the amounts and 109 concentrations used.

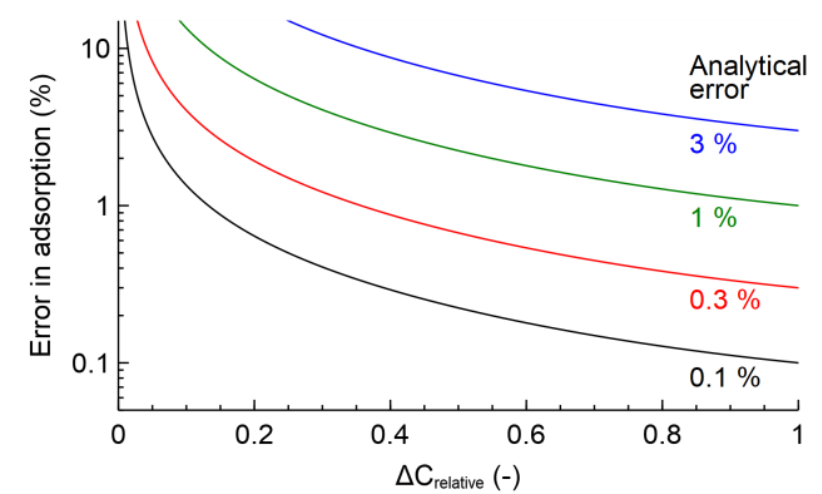

Fig. 1. The relative adsorption error as a function of analytical error and the relative change in adsorbate concentration; the analysis is based on the material balance approach (equation 1). 
114 The error analysis shows that there is a limit on the concentration range that could result in

115 accurate experimental results on adsorption. Moreover, excellent analytical reproducibility is

116 important being a limiting factor in adsorption accuracy.

117 Considering limitations in the material balance approach, an alternative method of directly

118 analysing the amount of material adsorbed seems particularly promising. Such a method

119 means detecting the adsorbed material directly on the adsorbent. In case of gas phase, such

120 studies are somewhat easy considering an absence of solvent and the possibility for direct

121 spectroscopic and even gravimetric analyses. Examples include titration of acid sides of

122 zeolite materials with nitrogen bases and observing a decrease in acidic groups in the

123 spectra or appearance of new bands [29,30]. These methods, however, are fraught with their

124 own issues and uncertainties [31]. (Spectroscopic studies require knowledge of molar

125 absorption coefficients which may change with coverage). Another way of studying the

126 amount adsorbed may be desorption experiments performed, for example, carrying out

127 solvent exchange [32] or temperature-programmed desorption [33].

128 The material balance experiments may over-estimate adsorption because of unforeseen

129 reactions between the adsorbate and the material; the desorption experiments under-

130 estimate in case of dissociative or strong adsorption. Therefore, all studies require attention

131 and care. Independent data validation such as a combination of both adsorption and

132 desorption or a combination of spectroscopy with gravimetry is a good way to minimise the 133 uncertainty [34].

3. Adsorption isotherms and data analysis

Once the adsorption data are obtained over a range of equilibrium concentrations, these are conventionally presented and analysed in terms of adsorption isotherms. There are many models that vary in physical meaning, the number of parameters and the accuracy of the description. Here, several widely used adsorption isotherm models are presented and more details could be found in reviews by Tran et al. or Rangabhashiyam et al. [17,26].

140 The Langmuir model is omnipresent because of simplicity and obvious physical meaning of

141 the parameters. Equation 4 shows the relationship between the adsorption at equilibrium

142 concentration $C_{\text {equilibrium }}$, adsorption constant $K_{L}$, and maximum adsorption capacity of $q_{\max }$ : 
144 The Langmuir model assumes that the adsorbent surface is uniform - it contains a fixed 145 number of sites that demonstrate identical adsorption energy, the adsorbant molecules could 146 adsorb reversibly only over unoccupied sites, and do not interact with each other.

147 Another widely used Freundlich isotherm is shown in equation 5:

$148 q_{e}=K_{F} C_{\text {equilibrium }}^{n}$,

149 where $K_{F}$ is the adsorption constant and $n$ is the empirical parameter. This model is

150 empirical; it often correctly describes adsorption over non-uniform surfaces but not the 151 saturation behaviour.

152 In performing a regression analysis of the experimental data, there are two potentially

153 conflicting aims: (i) to describe the system behaviour, or (ii) to gain mechanistic insights. The

154 mechanistic aim, obviously, requires rigour in the data analysis and independent validation

155 of the model assumptions. The descriptive aim seems deceptively simple. But the

156 descriptive models are often extrapolated beyond the studied range of parameters. In this

157 case, a careful analysis of the model and the data obtained becomes vital.

158 Correlation does not mean causation - people with umbrellas, although strongly correlating,

159 do not cause rain. A computationally accurate description of a particular isotherm (good fit)

160 does not show that the model is correct [31]. The model assumptions might not be fulfilled

161 resulting in unexpected behaviour beyond the studied range. Even if the model is correct,

162 the parameters obtained may be inaccurate resulting in unreliable extrapolations. Stitt at al.

163 [35] writes: "Just because the results are in colour, it doesn't mean they are right" and

164 provides ample examples of totally inaccurate yet computationally valid model descriptions.

165 A minor mistake in the model may lead to dramatic changes in the extrapolated results [35].

166 The example in Fig. 2 shows the results of kinetic modelling of the same data with various

167 models. All of these models showed an excellent fit with the $R^{2}$ values above $95 \%$, most

168 above 99\%. The dashed line shows the "correct" data possible because the fitted data were

169 artificially generated from a known kinetic model. Despite an excellent fit in all the models,

170 the results are dramatically different - as much as 350\% different! 


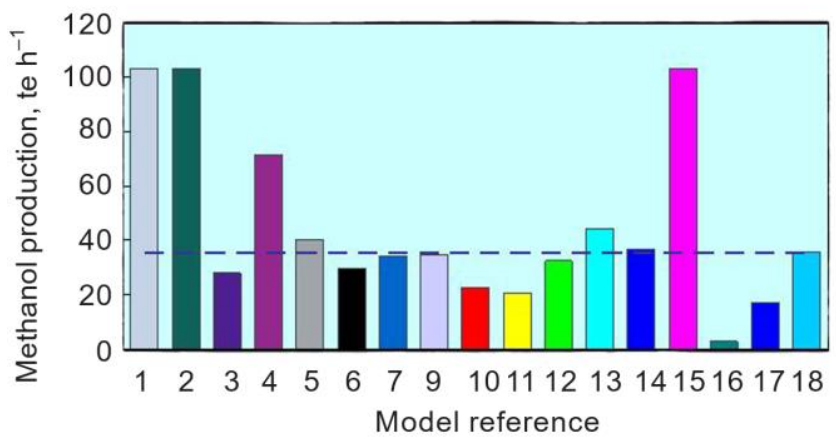

171

172 Fig. 2. The discrepancy in various kinetic models of the artificially generated kinetic data

173 (dashed line). Reprinted from ref. [35] which is based on the data obtained from ref. [36].

174

175 To avoid such poor results as in Fig. 2, the model must be validated by varying other

176 parameters such as temperature/pressure dependence. In the case of gas-phase

177 adsorption, Malek and Farooq [24] studied several models and compared the temperature

178 behaviour and accuracy in describing the competitive adsorption. But the first step in

179 assessing the model is to perform curve fitting and ensure that the parameters are well

180 defined.

181 The confidence intervals are most often estimated using black-box computational packages

182 that provide totally misleading data especially for non-linear models such as Langmuir

183 isotherm. A conventionally used linearization approach, on the other hand, introduces

184 additional errors and affects error estimates by imposing co-variation between parameters.

185 Bolster and Hornberger [37] discuss that Langmuir linearization may lead even to

186 computationally inaccurate results. A dramatic example is presented by Hamdaoui [38] who

187 applied two Langmuir linearization methods to the experimental data and obtained

188 dramatically different parameters. The differences between the Langmuir parameters

189 obtained with 2 models ranged between $-85 \%$ to $+27 \%$, while the goodness of fit was above

$19098.5 \%$ in both cases. Goodness of fit is not sufficient! 
213 Considering that in most cases, the relative experimental errors are constant (except when 214 close to the detection limit) [27,28], the objective function could be simplified as in equation 8 215

Fig. 3. Parameters of a Langmuir isotherm fitted with two linearization methods for two samples. The figure is based on the data by Hamdaoui [38].

Supplementary SI2 takes adsorption parameters from ref. [39], generates sets of model datapoints with an introduced normally-distributed error and performs regression analysis using various methods. Not surprisingly, the results show that the non-linear Langmuir approach with proper error weighing (discussed below) provide results closest to the correct adsorption parameters. Disregarding error weights creates almost twice larger deviations from the correct values, while linearization methods often produce unreliable data.

Proper curve fitting. The regression analysis has to be done properly as the example in Fig. 3 demonstrates. The first step is to avoid linearization and use non-linear model equation combined with the estimated experimental analytical errors. That may sound difficult but proper regression is simple - it minimises the objective function (weighed residual) as shown in equation 6 :

$$
f_{\text {objective }}=\sum_{i} \omega_{i}\left(q_{\text {experiment }, i}-q\left(C_{i}\right)\right)^{2},
$$

where $q_{\text {experiment, } i}$ are experimental adsorption datapoints, $q\left(C_{i}\right)$ the model values, and $\omega_{i}$ are the statistical weights of the $i$-th point. It is the weighing factor that makes a difference.

The weighting factors are calculated from the expected analytical uncertainties using equation 7, where $\sigma_{i}$ is the uncertainty (standard deviation) in the analysis of datapoint $i$, and $\xi_{i}$ is the relative experimental error (typically $0.5-2 \%=0.005-0.02$ ). A more general case of errors both in $\mathrm{X}$ and $\mathrm{Y}$ axes is shown in Supplementary SI1.

$$
\omega_{i}=1 / \sigma_{i}^{2}=1 /\left(\xi_{i} q_{\text {experiment }, i}\left(C_{i}\right)\right)^{2}
$$

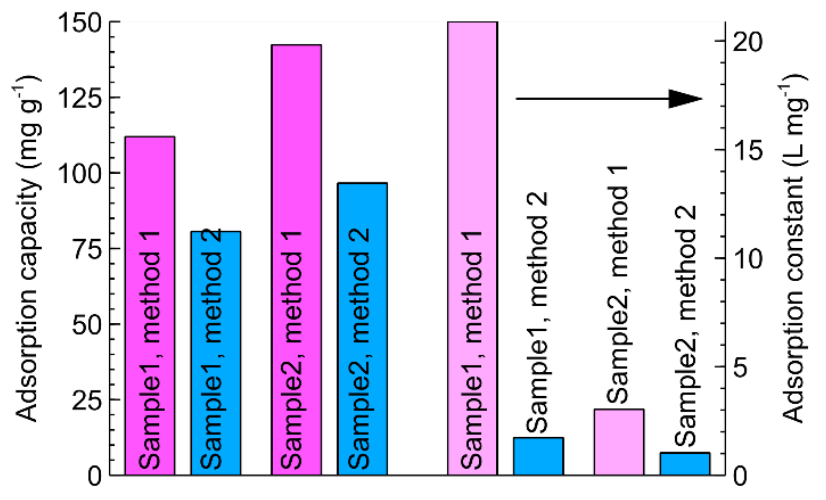


$216 \quad f_{\text {objective }}^{\prime}=\sum_{i}\left(1-\frac{q\left(C_{i}\right)}{q_{\text {experiment }, i}}\right)^{2}$.

217 Performing regression in most of the software packages (Origin, Excel) would result in the

218 implicit assumption of constant absolute errors (rather than relative ones). As a result, high

219 concentrations (with the corresponding high absolute errors) will be disproportionally well

220 fitted and low concentrations may be, in effect, neglected. This approach of constant

221 absolute errors combined with non-linear model may lead to worse results compared to

222 unpredictable linearized approaches. The most reliable approach, however, is to use non-

223 linear model with the objective function in equation 8. Supplementary SI2 elaborates on the

224 inaccuracy of linearization models.

225 Confidence intervals. Once a proper curve fitting is performed, the next step is to study the

226 confidence intervals. Likely the simplest and the most reliable way to perform such an

227 analysis is to use the Monte-Carlo method described by Alper and Gelb [40] - the method

228 that automatically accounts for co-variation in the model parameters. Many software

229 packages generate some estimations on the confidence intervals but these may be grossly

230 inaccurate [40].

231 The idea of the Monte-Carlo method is to generate a number (100-1000) of mock fitting data

232 (experimental data plus random noise with the experimental standard deviation). These

233 mock data are fitted and generate a set of model parameters. The statistics of the

234 parameters obtained show the confidence intervals.

235 Broad confidence intervals show that the values cannot be relied upon and the model likely

236 contains too many parameters. The widely used adsorption models contain only two

237 parameters, but there are many models with as much as 5 parameters [24,26,41]. A larger

238 number of parameters improves goodness of fit but makes all the parameters far less 239 defined.

4. Analysing thermodynamic parameters

241 Any spontaneous process occurs because the system moves toward the minimum of the

242 Gibbs's free energy, $\Delta \mathrm{G}^{\circ}$ ads in equation 9. Considering that the adsorbate molecules move

243 from the 3-dimentional freedom of the solution onto a 2-dimensional catalyst surface, the

244 adsorption entropy ( $\Delta \mathrm{S}^{\circ}$ ads) often decreases. In this case, the adsorption enthalpy $\left(\Delta \mathrm{H}^{\circ}\right.$ ads $)$

245 must be negative for the adsorption to take place.

$246 \Delta G^{o}{ }_{a d s}=\Delta H^{o}{ }_{a d s}-T \Delta S^{o}{ }_{a d s}$. 
247 The thermodynamic parameters of the adsorption are often obtained from the relation

248 between the adsorption constant $\left(K_{\text {equilibrium }}\right.$ in $\left.\mathrm{L} \mathrm{mol}^{-1}\right)$ and the Gibbs free adsorption energy

249 (van't Hoff equation) 10. Here $R$ is the universal gas constant and $T$ is the adsorption

250 temperature. The adsorption constant here could be either Langmuir of Freundlich constants

251 in case of diluted solutions. More details on the ways to obtain the constant suitable for this

252 equation are provided in reviews $[42,43]$.

$253 \Delta G_{a d s}^{o}=-R T \ln K_{\text {equilibrium }}$

The problem of the dimensioned constant. The Gibbs standard energy has dimensions of $\mathrm{J} \mathrm{mol}^{-1}$, the same as these of $R T$. Hence, the logarithm and the constant is dimensionless.

There is a controversy in how to resolve the misalignment of dimensions $-K_{\text {equlibrium }}$ must be dimensionless but the measured constant has the inverse units of concentration. There is a flurry of recent papers on the topic which argue on how to reconcile this problem of dimensions. First of all, both sides agree that the units of measured adsorption constant must be recalculated into $\mathrm{L} \mathrm{mol}^{-1}$, rather than keeping more exotic units such as $\mathrm{mg} \mathrm{g}^{-1}$.

261 Such a simple matter is sometimes overlooked rendering results incorrect $[26,42,44]$.

262 There is a hot disagreement, however, on the ways how to convert the dimensioned into the 263 dimensionless constant. The problem comes from the value of the equilibrium adsorption 264 constant shown in equation 11:

$K_{\text {experimental }}=\frac{\alpha_{A_{-} \text {adsorbed }}}{\alpha_{A_{-} \text {in_solution }} \alpha_{\text {adsorbent }}}$.

Here $K$ is th species: adsorbed compound $A$, compound $A$ remaining in the solution at equilibrium, and the adsorbent itself. The equation could be simplified considering activity coefficients 1 (which is valid for non-ionic and diluted ionic solutions $[42,45]$ ) and considering the Langmuir model the equilibrium coverage of $\theta$. The resulting equation 12 shows the origin of the

271 dispute - the constant determined experimentally has units of inverse concentration.

272

$$
K_{\text {experimental }}=\frac{\theta}{C_{A_{-} \text {in_solution }}(1-\theta)} \text {. }
$$

273 Both sides of the dispute agree that the problem shall be resolved by multiplying the 274 experimental constant (in $\mathrm{L} \mathrm{mol}^{-1}$ ) by a certain coefficient (in $\mathrm{mol} \mathrm{L}^{-1}$ ) that renders the product dimensionless. The value of the coefficient is disputed. 
276 On one side, Milonjić [46] and Tran et al. [26,45] suggests multiplication by $55.5 \mathrm{~mol} \mathrm{~L}^{-1}$. This 277 constant is a concentration of water in the solution. Zhou and Zhou [45] provide a detailed 278 proposition explaining that the constant of $55 \mathrm{~mol} \mathrm{~L}^{-1}$ comes from considering adsorption 279 competition between the adsorbate and the solvent.

280 On the opposite side, Liu [47], Ghosal and Gupta [42] provide reasons that the constants 281 shall be multiplied by $1 \mathrm{~mol} \mathrm{~L}^{-1}$. The reasoning comes from the description of thermodynamic 282 constants in terms of activities, not concentrations. The activity of component $A$ is shown in 283 equation 13:

284 $\alpha_{A}=\gamma_{A} \frac{C_{A}}{C_{r e f}}$,

285 where $\gamma_{A}$ is the activity coefficient, $C_{A}$ is the concentration of the adsorbed specie in mol $286 \mathrm{~L}^{-1}$, and $C_{r e f}$ is the concentration of the reference state equal to $1 \mathrm{~mol} \mathrm{~L}^{-1}[42]$.

287 The reader is advised to read papers from both sides of the argument (in particular papers 288 by Ghosal and Gupta [42], and Zhou and Zhou [45]) to form a coherent picture. In the 289 current review the author, however, offers additional reasoning that may clarify the dispute. 290 There are two points that both lead to the same conclusion on resolving the dispute.

291 The first point is that the same problem of dimensioned adsorption constants shown in 292 equation 12 equally applies to gas phase, but there is no disagreement there. The gas293 phase adsorbate concentrations may be presented in the units of in $\mathrm{mol} \mathrm{L}^{-1}$, or even mol $294 \mathrm{~mol}^{-1}$ (molar fraction of adsorbate in gas phase). More conventionally, however, pressure is 295 used as a measure of adsorbate activity rendering the corresponding experimental constant 296 in bar ${ }^{-1}$. This difference between the gas and liquid phases, however, shows that rendering 297 the concentration itself dimensionless (using mole fraction) makes little sense. In liquid 298 phase, multiplication of the constant by $55.5 \mathrm{~mol} \mathrm{~L}^{-1}$ renders, in effect, concentrations 299 dimensionless molar fractions. The reason is that using mole fractions does not explain the 300 increasing adsorption at a higher pressure. If we double pressure, we expect higher 301 adsorption. If we consider molar fraction (that is 1 regardless of pressure) we would struggle explaining the difference in adsorption. Hence, the experimentally determined adsorption constant must be dimensioned in gas and liquid phases. The second point comes from the analysis of how the problem of dimensioned constants could affect the resulting Gibbs energy assuming for the argument's sake the possibility of logarithmic Joule as a physical unit. Equation 14 explicitly states the constant $\vartheta$ required to make the experimental constant dimensionless: 
309 Considering the arithmetic of logarithms, equation 14 shows that such constant $\vartheta$ simply 310 provides an offset to the determined Gibbs energy of $-R T \ln \vartheta$. The corresponding offset 311 affects the absolute value of the Gibbs energy we observe. Considering equation 9, this 312 offset translates to an offset in the value of the adsorption enthalpy but does not affect the 313 adsorption entropy which depends on the slope in the $G$ vs $T$ plot.

314 This consideration implies that the absolute value of the Gibbs energy, as well as enthalpy, 315 cannot be determined, while that of entropy could be. Such a trivial statement,

316 unsurprisingly, agrees with thermodynamics - the existence of absolute values of entropy

317 and only relative values of enthalpy. For simplicity, enthalpy is often referred to certain 318 reference states as elements stable under normal temperature and pressure.

319 The standard conditions for gases, defined by IUPAC, are the temperature of $0{ }^{\circ} \mathrm{C}$ and a 320 pressure of 1 bar. Hence, all gas-phase adsorption constants are calculated in terms of bar ${ }^{-1}$ 321 with the (often implied) "dimensionalisation" constant $\vartheta$ of 1 bar [48]. In liquid phase, the 322 standard IUPAC state is for a solute at a concentration of $1 \mathrm{~mol} \mathrm{dm}^{-3}$ rendering the required 323 "dimensionalisation" constant $\vartheta$ of $1 \mathrm{dm}^{3} \mathrm{~mol}^{-1}$ [48]. Therefore, the correct way of calculating 324 the thermodynamic parameters from the liquid-phase adsorption data is shown in equation 325 15:

$\Delta G_{\text {ads }}^{o}=-R T \ln \left(K_{\text {experimental }}\left[\right.\right.$ in $L m^{-1}$ for diluted solutions $\left.]\right)$.

327 Analysis of adsorption thermodynamics. If the Gibbs adsorption energy obtained is 328 negative, the adsorption is often said to occur "spontaneously" [26,41,42,44,46]. However, 329 the "spontaneous" does not mean "occur". A small but negative value of the Gibbs free 330 energy shows that the equilibrium constant is below 1 (equation 10). This note may be of 331 little practical value for the design of pollutant adsorbents that must strongly adsorb to be 332 useful. For the catalytic applications, however, both too high and too low adsorption constants are obstacles for the reaction. An "optimal" adsorption constant (that provides the highest reaction rate) might be below 1 , the Gibbs adsorption energy may conceivably be positive. Having the Gibbs adsorption energy determined, the adsorption enthalpy and entropy may be obtained using equation 9 by studying adsorption at various temperatures. Such a derivation, albeit trivial, raises an interesting question of correlation between the parameters derived. Anastopoulos and Kyzas in their review [44] show a strong correlation between the thermodynamic parameters obtained in the literature (Fig. 4) and call for alternative and independent ways to validate the parameters. 


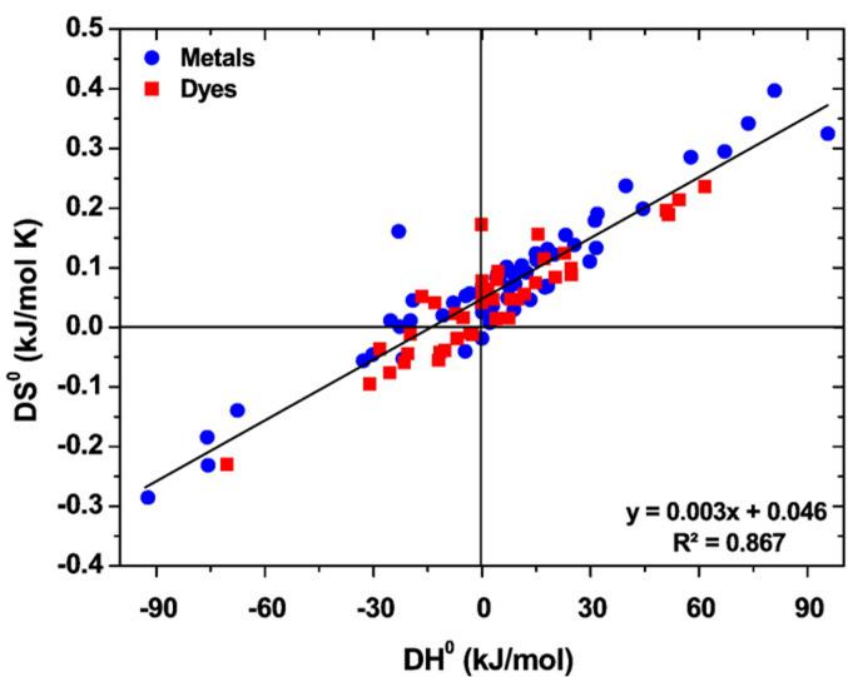

343 Fig. 4. Correlation between the adsorption entropy and enthalpy observed for the dyes and metal ions over various materials. The picture is reprinted from review [44].

345 The observed values of adsorption entropies and enthalpies can fall into 4 major groups

346 depending on if they are positive or negative. Fig. 4 shows that the more prevalent are cases

347 with exothermic adsorption with a negative adsorption enthalpy; and endothermic reactions

348 with the increasing entropy. However, all the cases are realised in the literature [41]

\section{Adsorption kinetics}

350 The adsorption kinetics are often studied using similar approaches as thermodynamics; the 351 only difference is the analysis in the adsorbate concentration change over time. The data 352 obtained are fitted to a model. Similarly to thermodynamics, fitting shall benefit from using 353 non-linearized equation to account for changing absolute experimental errors (and often 354 constant relative experimental errors). (Equation 8 provides the way to calculate fitting 355 residuals in such a case). The uncertainty intervals of the model parameters obtained shall 356 be checked using Monte-Carlo.

357 A particular consideration for kinetics is the required analysis duration. Fig. 5 shows 358 computer-generated adsorption data based on pseudo-second order kinetics with the 359 analytical uncertainty of $3 \%$ (Supplementary SI3). Once the equilibrium is reached, the 360 concentration does not change and the data points characterise the adsorption 361 thermodynamics, not kinetics. Hence, taking too many of these points into regression may 362 introduce unnecessary errors into the parameters determined; in the best case, these points 363 are valueless. 
364

365

366

367

368

369

370

371

372

373

374

375

376

377

378

379

380

381

382

383

384

385

386

387

388

389

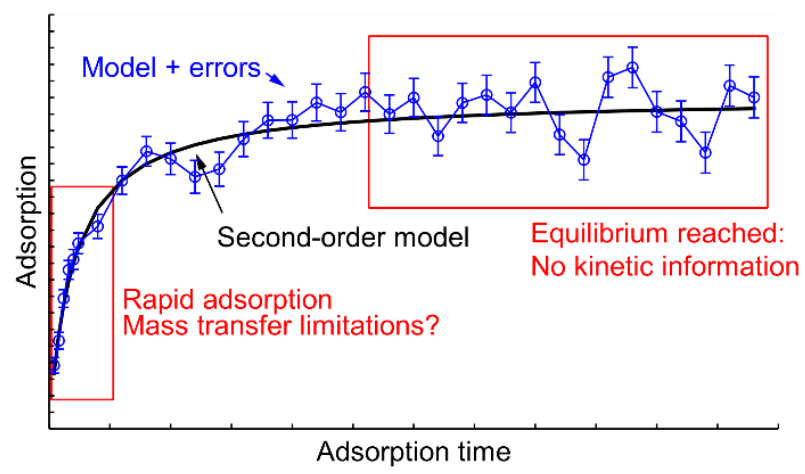

Fig. 5. Computer-generated data for pseudo-second order adsorption model with an experimental error at $3 \%$ (standard deviation) analytical uncertainty. The initial region may be limited by mass transfer; the equilibrium region contains no kinetic information.

While analysing adsorption kinetics, similar to reaction kinetics, one needs to study if the external mass transfer is not the rate-limiting factor. Otherwise, the data will reflect the rate of stirring rather than intrinsic adsorption. A simple way to check for external mass transfer is to perform experiments at various stirring rates and observe rates of adsorption constant above a certain stirring rate. However, many small reactors show minor changes in the Reynolds number in a broad stirring rate and may provide misleading results [49]; too high rotation rate, however, may decrease mass transfer [50]. Hence, a comparison of smaller and larger stirrers, possibly impellers, may be carried out for the test [51].

Internal mass transfer - the diffusion of the adsorbate molecules into the pores may often be a rate-limiting factor. In this case, the researcher may either look for the intrinsic rates of adsorption that requires studies with smaller adsorbent particles or using wall coatings $[14,52,53]$. Often, however, it is more practically important to characterise the apparent rates - in effect, internal mass transfer because such data characterises the behaviour of the adsorbents in many practical applications. The examples include adsorption of metal ions and dyes over chitosan (lobster shell waste) [21] and similar agricultural waste materials that have low porosity [32,54].

There are 3 most widely models used for describing the adsorption kinetics: pseudo first or second order, and internal diffusion. A wider range of adsorption models and equations are discussed by Qiu et al. [54].

Pseudo first or second order models treat adsorption in terms of a corresponding order chemical reaction with rate equations shown in equations 16 and 17 :

$\frac{d q(t)}{d t}=k_{1}\left(q_{\text {equilibrium }}-q(t)\right) ; q(t)=q_{\text {equilibrium }}\left(1-\exp \left(-k_{1} t\right)\right)$ 
$390 \frac{d q(t)}{d t}=k_{2}\left(q_{\text {equilibrium }}-q(t)\right)^{2} ; q(t)=\frac{q_{\text {equilibrium }}^{2} k_{2} t}{q_{\text {equilibrium }} k_{2} t+1}$,

391 where $k$ are apparent adsorption rate constants, $q(t)$ is the adsorption at the moment of $t$ 392 and $q_{\text {equilibrium }}$ is the equilibrium adsorption. These equations are often linearized, which as 393 discussed above, may introduce unpredictable errors into the parameters obtained by 394 regression (Fig. 3).

395 The other commonly used model considers intra-particle diffusion as the rate-limiting step.

396 The adsorbent is considered homogeneous spheres with the mass transfer determined by 397 equation $18[54,55]$ :

398

$\frac{\partial q(t, r)}{\partial t}=\frac{D_{s}}{r^{2}} \frac{\partial}{\partial r}\left(r^{2} \frac{\partial q(t, r)}{\partial r}\right)$

399 where $q$ is the adsorption at the moment $t$ and radial position in the particle $r, D_{s}$ is the

400 intra-particle diffusion coefficient. This equation could be solved and simplified to obtain

401 equation 19:

402

$q(t)=q_{\text {equilibrium }} \cdot 6 \sqrt{\frac{D_{s}}{\pi R^{2}}} \sqrt{t}$,

403

where $q_{\text {equilibrium }}$ is the equilibrium adsorption and $R$ is the particle radius. Such an

404

approximation is valid for $q(t) / q_{\text {equilibrium }}<0.3[54,55]$.

405

\section{Conclusions}

406 The review discusses experimental approaches, modelling to obtain thermodynamic, and

407 kinetic parameters of adsorption. The suggested workflow is schematically presented in Fig.

4086.

409 The planning steps involve assuming or estimating the adsorption constant; calculating the

410 adsorbent mass and adsorbate concentrations to ensure that the measured adsorption could

411 provide the desired accuracy. Reasonable accuracy is expected if the initial concentration

412 changes by at least $10 \%$ relative to the initial concentration; otherwise the experimental

413 errors would make results unusable (Fig. 1).

414 The experiment steps involve adsorption measurements and assessing the data with

415 alternative methods to exclude the possibility of false readings caused by side-phenomena

416 other than adsorption. An always advisable sanity check may involve comparing the 
417 adsorption data with the known properties of the material such as overall surface area or

418 adsorption site stoichiometry.

419 The modelling steps include selecting the adsorption model, curve fitting of the experimental 420 data using the original non-linear equations, and considering experimental errors. The fitting 421 objective function may be the one shown in equation 8 - the function that assumes constant 422 relative errors in adsorption and no errors in the $X$ axis values. Confidence intervals of the 423 model parameters shall be calculated for mathematical sanity of the model using the Monte-

424 Carlo approach [40]. Linearized models provide less predictable and often outright 425 inaccurate results as shown in Supplementary S12. Such calculations may be performed 426 using the Matlab code for the Langmuir model; a standalone executable freely available at 427 ref. [56].

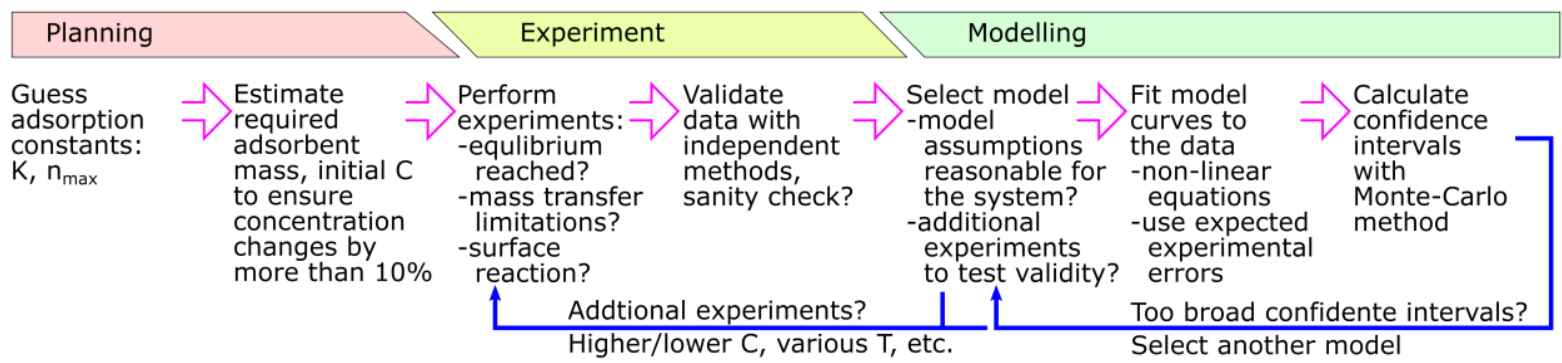

429 Fig. 6. Proposed workflow for adsorption experiments and analysis.

430 These steps allow obtaining mathematically reasonable results and assess the goodness of 431 fit for the model selected. However, a good fit of the model and narrow confidence intervals 432 do not validate the underlying model assumptions. If the modelling aims to probe into the 433 adsorption mechanisms, the model assumptions must be checked independently. If the 434 model aims only for a description of the experimental data, particular attention shall be given 435 on the range of studied conditions, extrapolation beyond the conditions would likely produce unreliable data.

\section{Acknowledgement}

438 The author is grateful for the support provided by IChemE Andrew Fellowship.

\section{References}

440 [1] UK Chemistry Growth | Overview, (2019). https://ukchemistrygrowth.com/overview/ $441 \quad$ (accessed May 8, 2019).

442 [2] H. Yi, M. Jiang, D. Huang, G. Zeng, C. Lai, L. Qin, C. Zhou, B. Li, X. Liu, M. Cheng, 443 W. Xue, P. Xu, C. Zhang, Advanced photocatalytic Fenton-like process over 444 biomimetic hemin-Bi2WO6 with enhanced pH, J. Taiwan Inst. Chem. Eng. 93 (2018) 184-192. doi:10.1016/j.jtice.2018.06.037. 
[3] Y. Yang, Z. Zeng, G. Zeng, D. Huang, R. Xiao, C. Zhang, C. Zhou, W. Xiong, W. Wang, M. Cheng, W. Xue, H. Guo, X. Tang, D. He, Ti3C2 Mxene/porous g-C3N4 interfacial Schottky junction for boosting spatial charge separation in photocatalytic H2O2 production, Appl. Catal. B Environ. 258 (2019). doi:10.1016/j.apcatb.2019.117956.

[4] H.-U. Blaser, Heterogeneous catalysis for fine chemicals production, Catal. Today. 60 (2000) 161-165. doi:10.1016/S0920-5861(00)00332-1.

[5] W.O. Haag, Catalysis by Zeolites - Science and Technology, Stud. Surf. Sci. Catal. 84 (1994) 1375-1394.

[6] P. Forzatti, L. Lietti, Catalyst Deactivation, Catal. Today. 52 (1999) 165-181. doi:10.1016/S0920-5861(99)00074-7.

[7] Z. Aksu, Application of biosorption for the removal of organic pollutants: A review, Process Biochem. 40 (2005) 997-1026. doi:10.1016/j.procbio.2004.04.008.

[8] A.J. Medford, A. Vojvodic, J.S. Hummelshøj, J. Voss, F. Abild-Pedersen, F. Studt, T. Bligaard, A. Nilsson, J.K. Nørskov, From the Sabatier principle to a predictive theory of transition-metal heterogeneous catalysis, J. Catal. 328 (2015) 36-42. doi:https://doi.org/10.1016/j.jcat.2014.12.033.

[9] J.K. Nørskov, T. Bligaard, B. Hvolbæk, F. Abild-pedersen, I. Chorkendorff, C.H. Christensen, The nature of the active site in heterogeneous metal catalysis, Chem. Soc. Rev. 37 (2008) 2163-2171. doi:10.1039/b800260f.

[10] V.D. Sokolovskii, Principles of Oxidative Catalysis on Solid Oxides, Catal. Rev. 32 (1990) 1-49. doi:10.1080/01614949009349939.

[11] J. Cheng, P. Hu, P. Ellis, S. French, G. Kelly, C.M. Lok, Brønsted - Evans - Polanyi Relation of Multistep Reactions and Volcano Curve in Heterogeneous Catalysis, J. Phys. Chem. C. 112 (2008) 1308-1311.

[12] M. Argyle, C. Bartholomew, Heterogeneous Catalyst Deactivation and Regeneration: A Review, Catalysts. 5 (2015) 145-269. doi:10.3390/catal5010145.

[13] N.C. Antonio J. Exposito, Yang Bai, Kirill Tchabanenko, Evgeny Rebrov, Process intensification of continuous flow imine hydrogenation in catalyst-coated tube reactors, Ind. Eng. Chem. (2019) submitted.

[14] Y. Bai, N. Cherkasov, S. Huband, D. Walker, R. Walton, E. Rebrov, Highly Selective Continuous Flow Hydrogenation of Cinnamaldehyde to Cinnamyl Alcohol in a Pt/SiO2 Coated Tube Reactor, Catalysts. 8 (2018) 1-18. doi:10.3390/catal8020058. 
[15] M. Besson, P. Gallezot, Deactivation of metal catalysts in liquid phase organic reactions, Catal. Today. 81 (2003) 547-559. doi:10.1016/S0920-5861(03)00153-6.

[16] M.A. Albiter, R.M. Crooks, F. Zaera, Adsorption of carbon monoxide on dendrimerencapsulated platinum nanoparticles: Liquid versus gas phase, J. Phys. Chem. Lett. 1 (2010) 38-40. doi:10.1021/jz900037x.

[17] S. Rangabhashiyam, N. Anu, M.S. Giri Nandagopal, N. Selvaraju, Relevance of isotherm models in biosorption of pollutants by agricultural byproducts, J. Environ. Chem. Eng. 2 (2014) 398-414. doi:10.1016/j.jece.2014.01.014.

[18] F. Naseeruteen, N.S.A. Hamid, F.B.M. Suah, W.S.W. Ngah, F.S. Mehamod, Adsorption of malachite green from aqueous solution by using novel chitosan ionic liquid beads, Int. J. Biol. Macromol. 107 (2018) 1270-1277. doi:10.1016/j.jijbiomac.2017.09.111.

[19] P. Nautiyal, K.A. Subramanian, M.G. Dastidar, Experimental Investigation on Adsorption Properties of Biochar Derived from Algae Biomass Residue of Biodiesel Production, Environ. Process. 4 (2017) 179-193. doi:10.1007/s40710-017-0230-2.

[20] R. Zambare, X. Song, S. Bhuvana, J.S. Antony Prince, P. Nemade, Ultrafast Dye Removal Using lonic Liquid-Graphene Oxide Sponge, ACS Sustain. Chem. Eng. 5 (2017) 6026-6035. doi:10.1021/acssuschemeng.7b00867.

[21] F.-C. Wu, R.-L. Tseng, R.-S. Juang, Kinetic modeling of liquid-phase adsorption of reactive dyes and metal ions on chitosan, Water Res. 35 (2001) 613-618. doi:10.1016/j.jcis.2005.01.093.

[22] R.L. Tseng, F.C. Wu, R.S. Juang, Liquid-phase adsorption of dyes and phenols using pinewood-based activated carbons, Carbon N. Y. 41 (2003) 487-495. doi:10.1016/S0008-6223(02)00367-6.

[23] X. Yang, B. Al-Duri, Kinetic modeling of liquid-phase adsorption of reactive dyes on activated carbon, J. Colloid Interface Sci. 287 (2005) 25-34. doi:10.1016/j.jcis.2005.01.093.

[24] A. Malek, S. Farooq, Comparison of Isotherm Models for Hydrocarbon Adsorption on Activated Carbon, AIChE J. 42 (1996) 3191-3201. doi:10.1002/aic.690421120.

[25] A. Henschel, I. Senkovska, S. Kaskel, Liquid-phase adsorption on metal-organic frameworks, Adsorption. 17 (2011) 219-226. doi:10.1007/s10450-010-9317-z.

[26] H.N. Tran, S.J. You, A. Hosseini-Bandegharaei, H.P. Chao, Mistakes and inconsistencies regarding adsorption of contaminants from aqueous solutions: A 

critical review, Water Res. 120 (2017) 88-116. doi:10.1016/j.watres.2017.04.014.

513 [27] J.C. Miller, J.N. Miller, Basic Statistical Methods for Analytical Chemistry, Analyst. 113

514

515

516

517

518

519

520

521

522

523

524

525

526

527

528

529

530

531

532

533

534

535

536

537

538

539

540

541

542

543

544 (1988) 1351-1356.

[28] J.N. Miller, Basic Statistical Methods for Analytical Chemistry Part 2, Analyst. 116 (1991) 3-14.

[29] M.A. Makarova, A.E. Wilson, B.J. van Liemt, C.M.A.M. Mesters, A.W. de Winter, C. Williams, Quantification of Brønsted Acidity in Mordenites, J. Catal. 172 (1997) 170177. doi:10.1006/jcat.1997.1849.

[30] D.B. Lukyanov, T. Vazhnova, N. Cherkasov, J.L. Casci, J.J. Birtill, Insights into Brønsted Acid Sites in the Zeolite Mordenite, J. Phys. Chem. C. 118 (2014) 2391823929. doi:10.1021/jp5086334.

[31] N. Cherkasov, T. Vazhnova, D.B. Lukyanov, Quantitative infra-red studies of Brønsted acid sites in zeolites: Case study of the zeolite mordenite, Vib. Spectrosc. 83 (2016) 170-179. doi:10.1016/j.vibspec.2016.02.002.

[32] V.K. Gupta, A. Mittal, V. Gajbe, Adsorption and desorption studies of a water soluble dye, Quinoline Yellow, using waste materials, J. Colloid Interface Sci. 284 (2005) 8998. doi:10.1016/j.jcis.2004.09.055.

[33] F. Salvador, M.D. Merchán, Study of the desorption of phenol and phenolic compounds from activated carbon by liquid-phase temperature-programmed desorption, Carbon N. Y. 34 (1996) 1543-1551. doi:10.1016/S0008-6223(96)00105-4.

[34] A.J. McCue, G.A. Mutch, A.I. McNab, S. Campbell, J.A. Anderson, Quantitative determination of surface species and adsorption sites using Infrared spectroscopy, Catal. Today. 259 (2016) 19-26. doi:10.1016/j.cattod.2015.03.039.

[35] B.H. Stitt, M. Marigo, S. Wilkinson, T. Dixon, How Good is Your Model ?, Johnson Matthey Technol. Rev. 59 (2015) 74-89. doi:10.1595/205651315X686804 JOHNSON.

[36] J.M. BERTY, S. LEE, F. SZEIFERT, J.B. CROPLEY, the "Uckron-1" Test Problem for Reaction Engineering Modeling, Chem. Eng. Commun. 76 (2007) 9-33. doi:10.1080/00986448908940315.

[37] C.H. Bolster, G.M. Hornberger, On the Use of Linearized Langmuir Equations, Soil Sci. Soc. Am. J. 71 (2007) 17961806. doi:10.2136/sssaj2006.0304er.

[38] O. Hamdaoui, Batch study of liquid-phase adsorption of methylene blue using cedar sawdust and crushed brick, J. Hazard. Mater. 135 (2006) 264-273. 
doi:10.1016/j.jhazmat.2005.11.062.

546

547

548

549

550

551

552

553

554

555

556

557

558

559

560

561

562

563

564

565

566

567

568

569

570

571

572

573

574

575

576

577

[39] Y.-T. Huang, M.-C. Shih, Effect of linearized expressions of Langmuir equations on the prediction of the adsorption of methylene blue on rice husk, Int. J. Sci. Res. Publ. 6 (2016) 549. www.ijsrp.org.

[40] J.S. Alper, R.I. Gelb, Standard errors and confidence intervals in nonlinear regression: comparison of Monte Carlo and parametric statistics, J. Phys. Chem. 94 (1990) 47474751. doi:10.1021/j100374a068.

[41] K.M. Doke, E.M. Khan, Adsorption thermodynamics to clean up wastewater; critical review, Rev. Environ. Sci. Biotechnol. 12 (2013) 25-44. doi:10.1007/s11157-0129273-z.

[42] P.S. Ghosal, A.K. Gupta, Determination of thermodynamic parameters from Langmuir isotherm constant-revisited, J. Mol. Liq. 225 (2017) 137-146. doi:10.1016/j.molliq.2016.11.058.

[43] H.N. Tran, S.J. You, H.P. Chao, Thermodynamic parameters of cadmium adsorption onto orange peel calculated from various methods: A comparison study, J. Environ. Chem. Eng. 4 (2016) 2671-2682. doi:10.1016/j.jece.2016.05.009.

[44] I. Anastopoulos, G.Z. Kyzas, Are the thermodynamic parameters correctly estimated in liquid-phase adsorption phenomena?, J. Mol. Liq. 218 (2016) 174-185. doi:10.1016/j.molliq.2016.02.059.

[45] X. Zhou, X. Zhou, The Unit Problem in the Thermodynamic Calculation of Adsorption Using the Langmuir Equation, Chem. Eng. Commun. 201 (2014) 1459-1467. doi:10.1080/00986445.2013.818541.

[46] S.K. Milonjić, A consideration of the correct calculation of thermodynamic parameters of adsorption, J. Serbian Chem. Soc. 72 (2007) 1363-1367. doi:10.2298/JSC0712363M.

[47] Y. Liu, Is the Free Energy Change of Adsorption Correctly Calculated?, J. Chem. Eng. Data. 54 (2009) 1981-1985. doi:10.1021/.je800661q.

[48] A.D. McNaught, A. Wilkinson, IUPAC. Compendium of Chemical Terminology 2nd ed. (the “Gold Book”), 1997. doi:10.1351/goldbook.I03352.

[49] R. Chambers, M. Boudart, Lack of Dependence of Conversion on Flow Rate in Catalytic Studies, J. Catal. 4 (1966) 141-145.

[50] I. Ayranci, S. Kresta, J. Shen, N. Semagina, Negative impact of high stirring speed in laboratory-scale three-phase hydrogenations, Ind. Eng. Chem. Res. 53 (2014) 
18091-18094. doi:10.1021/ie5017927.

579 [51] J. Hajek, D.Y. Murzin, Liquid-Phase Hydrogenation of Cinnamaldehyde over a Ru -

580

581

582

583

584

585

586

587

588

589

590

591

592

593

594

595

596 Sn Sol - Gel Catalyst . 1. Evaluation of Mass Transfer via a Combined Experimental / Theoretical Approach, Ind. Eng. Chem. Res. 43 (2004) 2030-2038. doi:10.1021/ie0340802.

[52] N. Cherkasov, Y. Bai, E. Rebrov, Process Intensification of Alkynol Semihydrogenation in a Tube Reactor Coated with a Pd/ZnO Catalyst, Catalysts. 7 (2017) 1-16. doi:10.3390/catal7120358.

[53] T. Omojola, N. Cherkasov, E. V. Rebrov, D.B. Lukyanov, S.P. Perera, Zeolite minilith: A unique structured catalyst for the methanol to gasoline process, Chem. Eng. Process. - Process Intensif. 131 (2018) 137-143. doi:10.1016/j.cep.2018.07.016.

[54] H. Qiu, L. Lv, B. Pan, Q. Zhang, W. Zhang, Q. Zhang, Critical review in adsorption kinetic models, J. Zhejiang Univ. A. 10 (2009) 716-724. doi:10.1631/jzus.a0820524.

[55] D.O. Cooney, Adsorption design for wastewater treatment, CRC Pres, INC., Boca Raton, Florida, USA. (1999).

[56] Adsorption Analysis - File Exchange - MATLAB Central, (n.d.). https://uk.mathworks.com/matlabcentral/fileexchange/72287-adsorption-analysis (accessed July 29, 2019). 